\title{
Percepção dos docentes sobre o ensino remoto em medicina durante a pandemia pela COVID-19
}

\author{
Teachers' perception of remote teaching in medicine during the pandemic by COVID-19 \\ Percepciones del profesorado sobre la enseñanza a distancia en medicina durante la pandemia \\ COVID-19
}

Recebido: 28/12/2021 | Revisado: 03/01/2022 | Aceito: 12/01/2022| Publicado: 14/01/2022

\author{
Larissa Cristina Machado de Barros \\ ORCID: https://orcid.org/0000-0002-2836-7707 \\ Centro Metropolitano da Amazônia, Brasil \\ E-mail: laridebarros@gmail.com \\ Márcia Bitar Portella \\ ORCID: https://orcid.org/0000-0002-4602-7618 \\ Centro Metropolitano da Amazônia, Brasil \\ E-mail: marciabitar@gmail.com \\ Deusa Meriam da Silva Brito \\ ORCID: https://orcid.org/0000-0001-9594-0385 \\ Centro Metropolitano da Amazônia, Brasil \\ E-mail: meriamsp@gmail.com \\ Adriana Letícia dos Santos Gorayeb \\ ORCID: https://orcid.org/0000-0003-3718-8417 \\ Centro Metropolitano da Amazônia, Brasil \\ E-mail: adriana@famaz.edu.br \\ Mariseth Carvalho de Andrade \\ ORCID: https://orcid.org/0000-0002-6257-5972 \\ Centro Metropolitano da Amazônia, Brasil \\ E-mail: marisethandrade1967@gmail.com
}

\begin{abstract}
Resumo
O presente estudo objetivou avaliar a percepção de docentes sobre o ensino remoto no curso médico durante a pandemia pelo SARS-CoV-2. Trata-se uma pesquisa de abordagem quantitativa com amostra de conveniência proveniente do quadro de docentes de uma escola médica privada. A população foi de 90 docentes que responderam ao questionário estruturado com escala Likert. Entre a amostragem, 47,8\% dos docentes afirmam dificuldades na adaptação para o ensino remoto emergencial, entretanto, 92,2\% deram importância ao treinamento institucional para as adaptações às metodologias ativas. A maioria considerou as atividades síncronas importantes, porém concordou que o ensino remoto gera desestímulo no discente, mas pode contribuir com o aprendizado, sem substituir as aulas presenciais. $\mathrm{O}$ aprendizado por meio remoto é possível e foi importante na pandemia, todavia o retorno das atividades presenciais é requisito fundamental para a formação médica.
\end{abstract}

Palavras-chave: Ensino; Estratégias educacionais; Educação médica; Metodologias ativas; Pandemia por COVID 19.

\begin{abstract}
This essay aims to assess the perception of professors about remote teaching in the medical course during SARSCoV-2 pandemic. This is a quantitative research approach where a convenience sampling was chosen taken from lectures of a private medical school. The population consisted of 90 professors who answered a structured questionnaire with Likert scale. Among the sample, $47.8 \%$ of professors stated difficulties in adapting to emergency remote teaching, however, $92.2 \%$ gave importance to institutional training for adaptations to active methodologies. Most of them considered synchronous activities important, although agreed that remote learning generates discouragement in students, but can contribute to learning, without substituting face-to-face tuition. Remote learning is possible and was important in the pandemic however, the return to face-to-face activities is a fundamental requirement for medical formation.
\end{abstract}

Keywords: Teaching; Educational strategies; Medical education; Active methodologies; Pandemic by COVID 19.

\section{Resumen}

El presente estudio tuvo como objetivo evaluar la percepción de los docentes sobre la enseñanza a distancia en el curso de medicina durante la pandemia SARS-CoV-2. Se trata de una investigación con enfoque cuantitativo con una muestra de conveniencia de la facultad de una facultad de medicina privada. La población estuvo conformada por 90 profesores que respondieron el cuestionario estructurado con una escala Likert. Entre la muestra, el 47,8\% de los 
docentes manifestó dificultades para adaptarse a la teleeducación de emergencia, sin embargo, el 92,2\% le dio importancia a la formación institucional para la adaptación a metodologías activas. La mayoría consideró importantes las actividades sincrónicas, pero coincidió en que el aprendizaje a distancia genera desánimo en los estudiantes, pero puede contribuir al aprendizaje, sin sustituir las clases presenciales. El aprendizaje a distancia es posible y fue importante en la pandemia, sin embargo, el regreso a las actividades presenciales es un requisito fundamental para la formación médica.

Palabras clave: Enseñanza; Estrategias educativas; Educación médica; Metodologías activas; Pandemia por COVID 19.

\section{Introdução}

A situação de pandemia pela COVID-19 e a necessidade de continuidade na formação médica foram fatores indutores para uma abrupta transição de atividades educacionais presenciais na educação médica para aprendizagem online. Este movimento foi marcado por adaptações ao ambiente virtual e criatividade para que estratégias fossem facilitadoras de aprendizagem por meio digital (Santos, 2020).

As Diretrizes Curriculares Nacionais (DCN) para os cursos de medicina de 2014 determinam o ensino presencial para a formação em saúde (Brasil, 2014). Porém, em março de 2020 no contexto da pandemia, o Ministério da Educação e Cultura MEC edita uma portaria que "Dispõe sobre a substituição das aulas presenciais por aulas em meios digitais enquanto durar a situação de pandemia do Novo Coronavírus" (Brasil, 2020).

Os docentes do curso de medicina do Centro Universitário Metropolitano da Amazônia - UNIFAMAZ receberam treinamento para que as atividades teórico-cognitivas fossem oferecidas aos alunos, por meio da plataforma Teams, na semana seguinte a publicação da portaria 343/2020 com a utilização das mesmas estratégias já desenvolvidas de forma presencial.

O curso de medicina do UNIFAMAZ possui currículo integrado, organizado por eixos temáticos que configuram um conjunto de unidades curriculares com atividades teóricas e práticas, distribuídos em 12 semestres e estágio curricular obrigatório em regime de internato nos quatro últimos semestres. O curso é desenvolvido com diversas estratégias de aprendizagem para grandes e pequenos grupos de alunos. No período anterior à pandemia da COVID -19, as estratégias aconteciam de forma presencial organizada por unidades curriculares. A plataforma Teams era utilizada por alguns docentes como meio de comunicação entre os alunos e para execução de algumas tarefas de forma assíncrona.

$\mathrm{O}$ impacto da rápida integração das estratégias educacionais às ferramentas digitais durante o momento pandêmico pode, de um lado, ter possibilitado novas oportunidades de aprendizado e de criatividade aos docentes, por outro lado, ter deixado dúvidas e incertezas de suas práticas, além de prováveis lacunas no aprendizado dos alunos, sobretudo nas atividades presenciais de desenvolvimento de habilidades.

Portanto, questiona-se como o docente de medicina de uma instituição de ensino do Norte do Brasil percebeu o processo de ensino-aprendizagem com a transição das ferramentas e estratégias metodológicas do ensino presencial para remoto no período da pandemia da COVID-19? Com base nisso, o presente estudo objetivou avaliar a percepção de docentes sobre o ensino remoto em medicina durante a pandemia pela COVID-19.

\section{Metodologia}

Trata-se de um estudo transversal, com abordagem quantitativa, por meio da análise de conteúdo proposta por Bardin (2011), desenvolvido no curso de medicina do Centro Universitário Metropolitano da Amazônia - UNIFAMAZ, uma IES privada, localizada no município de Belém, Estado do Pará.

A população de estudo foi composta pelos docentes que atuaram do $5^{\circ}$ ao $11^{\circ}$ período do curso durante a pandemia da COVID-19. Compõem o total da população alvo da pesquisa 118 docentes, considerado $\alpha=5 \%$, sendo estatisticamente significante; o cálculo para amostra de conveniência proveniente de população fechada contou com 90 docentes selecionados, 
que responderam ao questionário da pesquisa.

Quanto aos critérios de inclusão e exclusão, foram incluídos os docentes que participaram das atividades acadêmicas teóricas e/ou práticas do curso de medicina em pelo menos dois períodos anteriores à pandemia pela COVID-19, e permaneceram em atividades acadêmicas no curso, nos diferentes contextos epidemiológicos vivenciados no cenário pandêmico. Excluíram-se os docentes regularmente vinculados a Instituição de Ensino Superior (IES), que se afastaram no período estudado e aqueles que não vivenciaram o momento anterior à pandemia.

A coleta e análise de dados foi desenvolvida por meio das etapas descritas a seguir: Na primeira Etapa, os docentes foram convidados a participar do estudo por meio de um questionário estruturado, em plataforma virtual Microsoft Forms $365 \circledR$. Este foi construído pelos pesquisadores utilizando a escala de Likert em cinco níveis de concordância e composto de questões objetivas sobre a percepção do público-alvo acerca das estratégias educacionais adaptadas no período da pandemia. As perguntas foram elaboradas e direcionadas tomando como base um ciclo de reuniões realizadas entre a gestão do curso e os docentes. Nas reuniões, foi solicitado aos docentes descreverem as experiências com as metodologias e as ferramentas utilizadas antes e durante o período pandêmico.

A segunda etapa foi de análise das respostas ao questionário aplicado, comparando as percepções entre as variáveis respondidas. Os dados foram categorizados, tratados por análise estatística para a avaliação quantitativa e inseridos em uma planilha eletrônica para procedimentos das análises descritivas e inferenciais. Para a análise dos resultados sobre a percepção dos docentes, as cinco opções de resposta foram inseridas em banco de dados Excel com agregação das alternativas discordo totalmente e discordo em "discordaram", assim como as respostas de concordo totalmente e concordo, agregados em "concordaram". Os resultados nem concordo e nem discordo foram considerados "neutros". As informações da caracterização amostral foram apuradas através do Google FORMS e convertidas para o software Microsoft $\circledast$ Office Excel® 2016. Na aplicação da Estatística Descritiva, foram construídos tabelas e gráficos para apresentação dos resultados e calculadas as medidas de posição e dispersão. A estatística analítica foi utilizada para avaliar os resultados das variáveis categóricas da amostra através dos Testes G e Qui-Quadrado aderência para tabelas univariadas e Independência, para análises bivariadas. As estatísticas descritiva e analítica, foram realizadas no software BioEstat ${ }^{\circledR}$ 5.4. Para a tomada de decisão, foi adotado o nível de significância $\alpha=0,05$ ou $5 \%$, sinalizando com asterisco $(*)$ os valores significantes.

A fim de garantir os aspectos éticos, foram respeitadas as Normas de Pesquisa Envolvendo Seres Humanos do Conselho Nacional de Saúde do Ministério da Saúde. Este estudo foi aprovado pelo Comitê de Ética em Pesquisa com seres humanos (CEP) do UNIFAMAZ, CAAE: 47817321.0.0000.57.01.

\section{Resultados e Discussão}

Dos 90 participantes que responderam ao questionário, 65.6\% (n=59) eram femininos, com diferença significativa entre a variável sexo. A média de idade dos participantes correspondeu a 43,9 anos, verifica-se que a maioria 60\% ( $\mathrm{n}=54$ ) é formada por docentes com formação profissional em Medicina, com tempo médio de formação de 19,6 anos, 88,9\% ( $\mathrm{n}=80$ ) deles com título stricto sensu e 19 anos de experiência de trabalho (Tabela 1). 
Tabela 1 - Correlação do uso das novas ferramentas virtuais de aprendizagem e as variáveis gênero, titulação, faixa etária e tempo de serviço.

\begin{tabular}{|c|c|c|c|c|c|c|c|c|}
\hline & $\mathbf{N}$ & \multicolumn{2}{|c|}{ Concordam } & \multicolumn{2}{|c|}{ Neutros } & \multicolumn{2}{|c|}{ Discordam } & $\begin{array}{l}\text { p-valor } \\
\text { 0.0470* }\end{array}$ \\
\hline Feminino & 59 & 24 & $40,7 \%$ & 3 & $5,1 \%$ & 32 & $54,2 \%$ & \\
\hline Masculino & 31 & 19 & $61,3 \%$ & 2 & $6,5 \%$ & 10 & $32,3 \%$ & \\
\hline Titulação & & & & & & & & 0,8559 \\
\hline Mestrado & 50 & 23 & $46,0 \%$ & 2 & $4,0 \%$ & 25 & $50,0 \%$ & \\
\hline Doutorado & 30 & 14 & $46,7 \%$ & 2 & $6,7 \%$ & 14 & $46,7 \%$ & \\
\hline Faixa etária (anos) & & & & & & & & 0.0012* \\
\hline 30 a 39 & 35 & 15 & $42,9 \%$ & 4 & $11,4 \%$ & 21 & $60,0 \%$ & \\
\hline 40 a 49 & 24 & 11 & $45,8 \%$ & 2 & $8,3 \%$ & 11 & $45,8 \%$ & \\
\hline 50 a 59 & 14 & 9 & $64,3 \%$ & 1 & $7,1 \%$ & 4 & $28,6 \%$ & \\
\hline$>=60$ & 12 & 8 & $66,7 \%$ & 0 & $0,0 \%$ & 4 & $33,3 \%$ & \\
\hline Mín / Média / Máx & & \multicolumn{2}{|c|}{$28-46.6-70$} & & $40.2-52$ & \multicolumn{2}{|c|}{$27-41.8-67$} & \\
\hline Tempo de serviço & & & & & & & & 0.0049* \\
\hline$<10$ & 15 & 7 & $46,7 \%$ & 2 & $13,3 \%$ & 6 & $40,0 \%$ & \\
\hline 10 a 19 & 39 & 14 & $35,9 \%$ & 1 & $2,6 \%$ & 24 & $61,5 \%$ & \\
\hline 20 a 29 & 17 & 9 & $52,9 \%$ & 1 & $5,9 \%$ & 7 & $41,2 \%$ & \\
\hline$>=30$ & 19 & 13 & $68,4 \%$ & 1 & $5,3 \%$ & 5 & $26,3 \%$ & \\
\hline Mín / Média / Máx & & \multicolumn{2}{|c|}{$05-22.0-45$ anos } & & $0-30$ anos & \multicolumn{2}{|c|}{$05-17.8-42$ anos } & \\
\hline
\end{tabular}

*Teste Qui-Quadrado; **Teste G Aderência. Fonte: Elaborado pelos autores.

Em relação à amostragem quanto a prática docente em tempos de pandemia pelo SARS-CoV-2, por meio do questionário, a Figura 1 evidencia que a afirmativa "o uso das novas ferramentas virtuais de aprendizagem teve difícil adaptação para docentes" resultou em relatos parcialmente semelhantes entre aqueles que discordam 46,7\% (n=42) e concordam, 47,8\% (n=43). Segundo Shuhmaker (2017), pesquisas realizadas em países que já resolveram problemas estruturais das escolas em relação as TICs com apoio técnico e pedagógico, ainda apresentam dificuldades quanto a integração dessas ferramentas na prática docente, admitindo-se que existem barreiras maiores que as identificadas, que resultam do fato de que alguns docentes não introduziram o computador na sua realidade e sentem-se constrangidos pela obrigação de usar a informática como instrumento de trabalho, e, Fonseca (2008) cita a pedagogia de Bachelard, afirmando que existe uma barreira epistemológica ao avanço tecnológico ou à mudança que gera estagnação e inércia. No estudo qualitativo de Modelski (2019), os depoimentos mostram que a familiaridade com as tecnologias virtuais decorre do uso contínuo pelo docente e que os investimentos na formação docente são insuficientes para quem está habituado à educação pouco interativa; que a tecnologia é uma ferramenta cultural e quanto maior a fluência no seu uso, mais tranquilidade o docente demonstra para criar possibilidades na sua prática pedagógica. Na análise da correlação entre o gênero, faixa etária, tempo de serviço e dificuldade de adaptação às novas ferramentas virtuais de aprendizagem foi encontrada diferença estatisticamente significante, ou seja, os homens encontraram mais dificuldades que mulheres, quanto maior a faixa etária, mais difícil a adaptação. Assim como os docentes com maior tempo de serviço. 
Figura 1 - Resultado sobre o uso das novas ferramentas virtuais de aprendizagem de acordo com a amostragem do estudo.

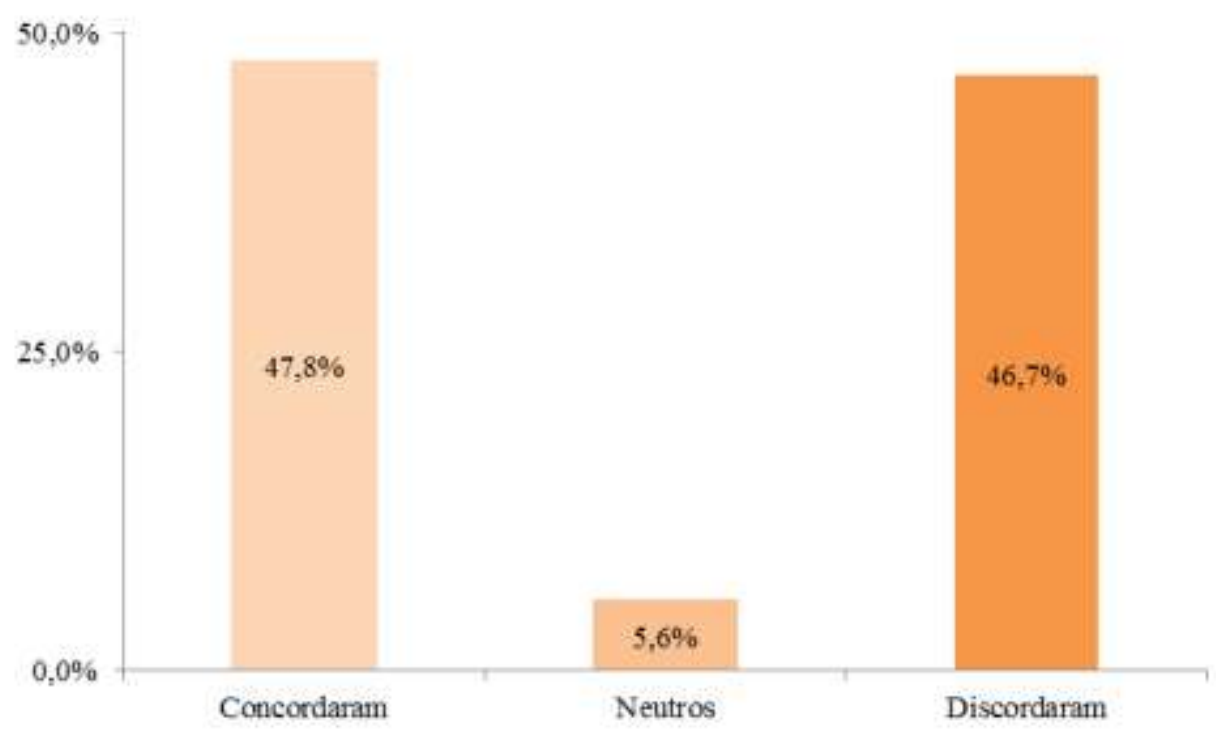

Fonte: Elaborado pelos autores.

A pandemia da COVID-19 causou uma ruptura abrupta nas rotinas de vida e trabalho em todo o mundo, obrigando a todos mudarem seu "status quo" e, no caso dos docentes, tiveram um exíguo tempo de treinamento e adaptação para enfrentar a nova realidade. Segundo Shuhmaker4, quando o professor não é capaz de dominar o processo metodológico pode cometer erros didáticos que irão influenciar na adesão e compreensão do aluno. Os resultados deste estudo são semelhantes ao de Shuhmaker4, onde menos de 50\% dos participantes usavam as TICs e chegavam a referir desconforto quando eram chamados a inserir as mesmas nos currículos.

Quanto às afirmativas "o treinamento institucional para a adaptação da metodologia ativa em meio remoto foi muito importante para seu desenvolvimento como docente na pandemia", assim como "a plataforma Teams" foi a grande facilitadora como ferramenta para o processo ensino-aprendizagem, obtiveram-se resultados semelhantes, salientado na Figura 2. Metade dos docentes participantes tinham habilidades tecnológicas e experiência com a utilização de estratégias educacionais em meio remoto, fato que podemos relacionar com a faixa etária destacada no perfil (30 a 39 anos), semelhante ao estudo de revisão bibliográfica de Santos et al. (2021), que lembra que os docentes nessa faixa etária cresceram com a evolução da internet. 
Figura 2 - A importância do treinamento institucional para a adaptação da metodologia ativa, e o uso da plataforma Teams pelos docentes na pandemia.

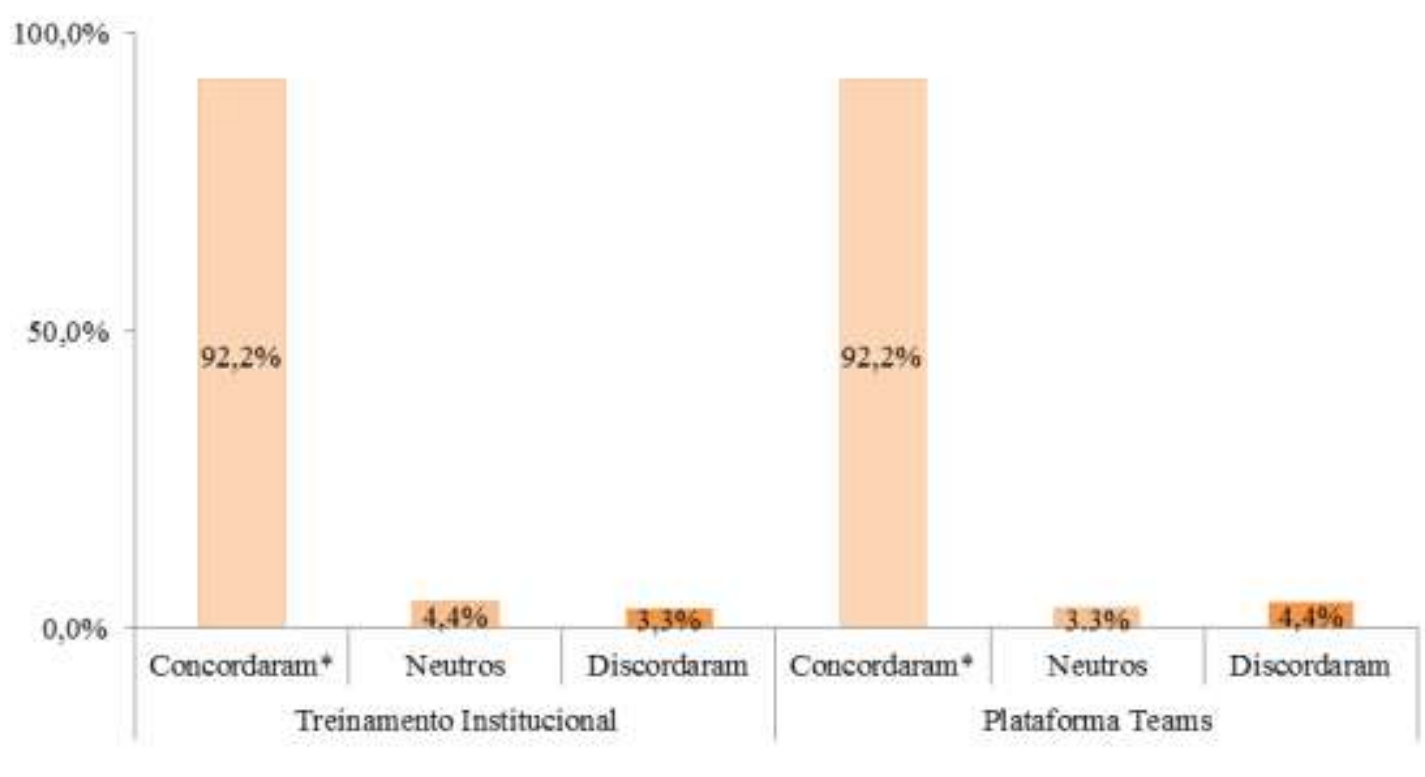

Fonte: Elaborado pelos autores.

A IES capacitou os docentes para o uso da Plataforma Teams, logo após a publicação da portaria MEC 343/2020, que permitiu a substituição das aulas presenciais por aulas online e 87,8\% $(n=79)$ participantes concordaram que esse treinamento facilitou o trabalho docente com os alunos, resultado semelhante ao de Burci et al. (2020) relatam que os ambientes virtuais de aprendizagem foram ferramentas que contribuíram com organização docente e gestora no momento de adequação a um novo cenário. Como na experiência da Faculdade de Ciências Médicas e da Saúde (FMCS-PUC) relatada por Mello et al. (2021), o curso de medicina da UNIFAMAZ utiliza metodologias ativas de ensino-aprendizagem e desenvolve atividades em pequenos grupos, com práticas de habilidades em laboratórios e comunidade e, conseguiu se organizar para atividades online com a utilização da Plataforma Teams da Microsoft em uma semana após a suspensão das aulas presenciais, entretanto, o grande desafio foi adaptar metodologias ativas para o meio remoto.

$\mathrm{Na}$ Tabela 2, o estudo mostra que a maioria dos docentes $71,1 \%,(\mathrm{n}=64)$ afirma que o aprendizado por meio remoto gera maior desestímulo nos módulos iniciais do curso, com proporção estatisticamente significativa entre os que concordam p<0,0001, corroborando o levantamento de Appenzeller et al. (2020) que aponta maior proporção de alunos com dificuldade de acesso nos anos iniciais de medicina, e ainda a dificuldade de acompanhar as aulas por computadores e notebooks compartilhados com outros membros da família. Silva et al. (2021) ao realizar uma revisão integrativa sobre a implantação de aulas remotas no curso de medicina, relata que as atividades online distanciam o professor do aluno, fragilizando o vínculo que é muito importante nos cursos de saúde, onde precisa ser desenvolvido de forma enfática a empática.

Tabela 2 - Aprendizado por meio remoto sobre desestímulo nos módulos iniciais do curso.

\begin{tabular}{cccc}
\hline $\begin{array}{c}\text { O aprendizado por meio remoto sofre maior desestímulo nos módulos iniciais } \\
\text { curso. }\end{array}$ & do \\
\hline \hline Concordaram* & 64 & $71.1 \%$ \\
Neutros & 8 & $8.9 \%$ \\
Discordaram & 18 & $20.0 \%$ \\
\hline
\end{tabular}

$*_{\mathrm{p}}<0.0001$ Teste Qui-Quadrado Aderência. Fonte: Elaborado pelos autores. 
O aluno ingressante ainda nem entendeu como estudar, ou desenvolver uma rotina de estudos e já teve que se adaptar ao desconhecido, muitas vezes confuso na sua organização mental para aprender a ser médico, conforme ratifica Rios (2021) os acadêmicos ingressantes no curso médico, têm potencialmente mais propensão à desorientação e ao estresse, e que, após o ingresso, tiveram que rapidamente se adaptar a metodologia e ao ensino remoto. Em seu estudo aponta que entre os alunos ingressantes, o tema recorrente está atrelado ao sentimento de medo: de ter mau desempenho nas provas, de perder o semestre e de não aprender. A percepção dos docentes em relação ao desestímulo nos períodos iniciais do curso pelos discentes pode estar associada à frustação das expectativas de quem entra em um curso de medicina ansioso pela prática com pacientes, de apreender procedimentos médicos, de estar nos laboratórios de anatomia e fisiologia ou morfofuncional. Como relata Gomes et al. (2020) quanto as expectativas nos anos iniciais do curso estão predominantemente associadas a inserção em contextos práticos, na atuação no processo saúde-doença em diferentes níveis de atenção, recuperando e reabilitando a saúde em todos os âmbitos e, à perspectiva de se tornar um egresso multidisciplinar, a partir da interação ensino-serviço e comunidade.

Todas essas premissas estão de acordo com as DCN para o curso de medicina como essenciais na formação médica, posto que, durante a formação, o ambiente de prática é insubstituível para o aperfeiçoamento de competências e, depois de formado, o médico deverá ser capaz de considerar o paciente em todas as suas particularidades, respeitando os direitos humanos e as diversidades culturais (Meireles et al., 2019).

Ainda sobre o ensino remoto, a maioria dos docentes $84,4 \%(n=76)$ discordam que a aprendizagem desse modo sofre as mesmas interferências dos alunos que a aprendizagem presencial (Tabela 3). Tais achados são respaldados por Santos1 que aponta os docentes como atores que reconhecem os processos de ensino-aprendizagem, pela sua particular característica de exigirem simultaneidade, que difere da sincronidade e, portanto afirmam que experienciar aprendizagens significativas só é possível, por meio da interação pessoal, da troca de conhecimentos, da expressão e partilha de sentimentos e emoções as quais são distorcidas pelo ensino, criando-se os "monólogos digitais". Para muitos destes docentes, o ensino em contexto de sala de aula é uma parte primordial da experiência de aprendizagem.

Tabela 3 - Percepção quanto as interferências na aprendizagem remota e presencial.

\begin{tabular}{ccc}
\hline A aprendizagem via remota sofre as mesmas interferências da atividade & \multicolumn{2}{c}{ presencial. } \\
\hline \hline Concordaram & 23 & $25.6 \%$ \\
Neutros & 1 & $1.1 \%$ \\
Discordaram* & 76 & $84.4 \%$ \\
\hline
\end{tabular}

*p $<0.0001$ Teste Qui-Quadrado Aderência. Fonte: Elaborado pelos autores.

Além disso, há uma grande disparidade e um complexo paradoxo social entre os antagonismos do homeoffice e a vida real (Santos, 2021). Dessa forma, o isolamento social e a adaptação do trabalho em casa, bem como a introdução das tecnologias na intimidade dos lares, têm causado uma sensação de perda da vida privada dos professores. A rotina online foi se sobrepondo a rotina offline ao passo que não se identifica onde termina uma e começa outra. Hábitos diários têm sido amplamente alterados e isso compromete significativamente o desempenho dos docentes que precisaram se adaptar a esta nova realidade (Saraiva et al., 2020).

Nos resultados detalhados na Tabela 4, os docentes afirmaram que a utilização de metodologias ativas no ensino remoto, contribuiu para o aprendizado dos discentes na pandemia 74,4\% ( $\mathrm{n}=64)$, semelhante aos estudos de Fossa et al. (2020), que investigaram as opiniões dos estudantes acerca desses recursos e metodologias educativas, demonstrando que os alunos aprovaram as adaptações realizadas às estratégias de ensino implantadas durante o isolamento social, estratégias essas, que mesmo familiares a muitos alunos, por utilizarem tecnologias digitais, não fazem parte do ensino médico tradicional. 
Tabela 4 - Contribuição da metodologia ativa no ERE para ao aprendizado discente.

\begin{tabular}{|c|c|c|}
\hline \multicolumn{3}{|c|}{$\begin{array}{l}\text { A utilização de metodologias ativas no ensino remoto, mesmo que na modalidade síncrona, } \\
\text { pouco contribui para o aprendizado discente. }\end{array}$} \\
\hline Concordaram & 18 & $20.0 \%$ \\
\hline Neutros & 5 & $5.6 \%$ \\
\hline Discordaram* & 67 & $74.4 \%$ \\
\hline
\end{tabular}

*p $<0.0001$ Teste Qui-Quadrado Aderência. Fonte: Elaborado pelos autores.

Neste sentido, a pesquisa de Faria e Amaral (2021), que utilizou três estratégias ativas de aprendizagem para o ensino de pediatria, concluiu que estas estratégias ativas podem ser adaptadas e possibilitam melhorias na qualidade do estudo remoto emergencial (ERE). No entanto, os resultados apresentados neste estudo inferem que nem todas as atividades práticas podem ser adaptadas ao meio remoto e podem deixar lacunas na aquisição de competências como habilidades e atitudes.

As estratégias e ferramentas metodológicas ativas favorecem a participação constante dos alunos de forma síncrona, assim como na elaboração de atividades colaborativas. Neste estudo 77,8\% (n=70) discordam que "todas as estratégias educacionais podem ser aplicadas via remota" e 67,8\% $(\mathrm{n}=61)$ concordaram que a distância professor- aluno trouxe prejuízo para o aprendizado (Figura 3). O ensino presencial possui logística, estratégias de aprendizagem já validadas e difundidas. As estratégias adaptadas ao ensino remoto passam a demandar outras variáveis, que podem não atender aos objetivos de uma dinâmica à distância como as atividades práticas, que demandam aquisição de habilidades e de atitudes nem sempre alcançáveis pelo docente durante o ensino remoto, mesmo síncrono (Silva et al., 2021b).

Figura 3 - Percepção dos docentes quanto às estratégias educacionais no processo de ensino-aprendizagem.

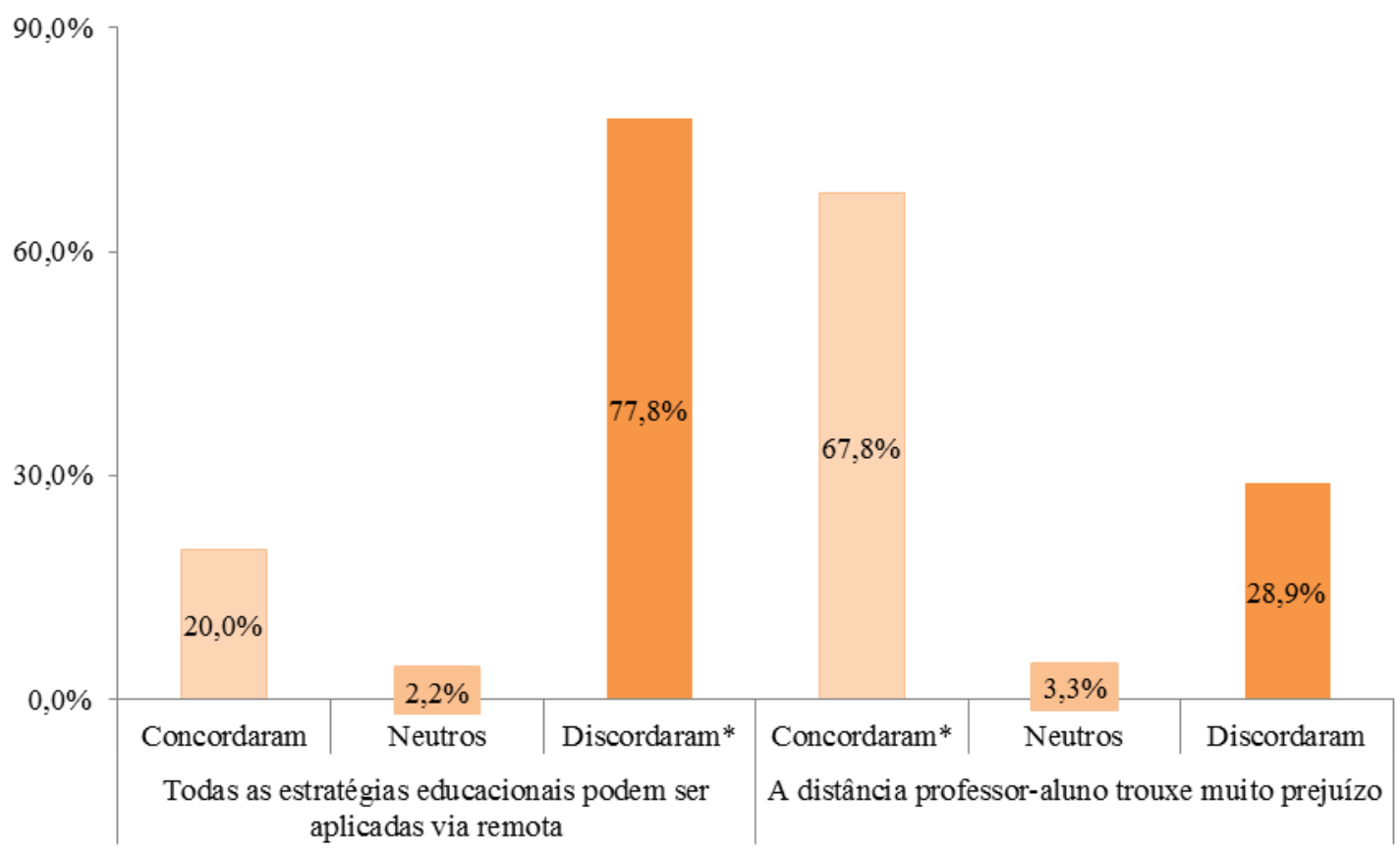

Fonte: Elaborado pelos autores.

A discordância quanto a aplicação de todas as estratégias educacionais via remota pode estar associada à condição do docente não se sentir plenamente habilitado ao uso das ferramentas, e pela dificuldade de acesso ou de concentração dos alunos, além do fato de que estudantes de medicina aguardam o atendimento do paciente como fonte de aprendizado porque o professor é modelo. Silva et al. (2021b), na sua revisão integrativa sobre "os desafios da implantação de aulas remotas no curso 
de medicina" relata que a formação em saúde tem estreita ligação com o ensino-serviço-comunidade e que um dos desafios é promover a adesão do docente ao processo tecnológico visando superar o distanciamento. Ainda, este autor relata que as principais críticas dos estudantes, já obtidas em estudos sobre o assunto, envolvem a demora ou a ausência de feedback pelos professores sendo esse um fator desmotivador para o aluno. Para César et al. (2020) no seu relato de experiência, as metodologias ativas podem ser realizadas de maneira remota, pois constituem mudança no campo educacional e que devem ser repensadas a partir desse momento.

A Tabela 5 demonstra que 73,3\% (n=66) dos participantes concordam que o ambiente doméstico influi negativamente no aprendizado com a dificuldade de muitos alunos, com proporção estatisticamente significante, principalmente das mulheres, citadas por alguns autores de compatibilizar as situações domésticas de trabalho com a família às necessidades do estudo, assim como o fato que os cenários domésticos nem sempre estão compatíveis com o ensino-aprendizagem e, essas interferências são bem diferentes no método presencial, onde o aluno está em um ambiente adequado que propicia maior capacidade de concentração. Marsilli et al., (2020), em sua revisão sistemática realizada com o objetivo de avaliar as experiências do ensino remoto pelo docente, considera que grande parte dos professores sentiu-se pressionada a assumir as responsabilidades pelo ensino remoto sem apoio técnico especializado e com a mudança teve que praticamente dobrar sua carga horária de trabalho influenciando na questão domestica sem contar coma pressão da própria pandemia sobre a família.

Tabela 5 - O ambiente doméstico interfere negativamente no aprendizado.

\begin{tabular}{ccc}
\hline O ambiente doméstico interfere negativamente no aprendizado. & \\
\hline \hline Concordaram* & 66 & $73.3 \%$ \\
Neutros & 10 & $11.1 \%$ \\
Discordaram & 14 & $15.6 \%$ \\
\hline
\end{tabular}

${ }^{*} \mathrm{p}<0.0001$ Teste Qui-Quadrado Aderência. Fonte: Elaborado pelos autores.

Sobre os tipos de estratégias de aprendizagem, similação realística e reunião de grupos, respectivamente, 75,6\% $(n=68)$ dos docentes concordaram que estas estratégias são comprometidas quando não realizadas de forma presencial (Tabela 6). Achado correspondente a pesquisa de Rodrigues et al. (2020) aponta que em resposta aos estágios cancelados, como ocorreu desde o início de 2020, muitas escolas Médicas, fizeram a transição para aulas em vídeo e salas de bate-papo online para substituir experiências clínicas. Embora a simulação tenha subsidiado oportunidades alternativas, percebeu-se uma falta de fidelidade e realismo com esses métodos de aprendizagem. Como resultado, esses estudantes de Medicina tiveram oportunidades reduzidas de aprendizado prático.

Tabela 6 - As atividades de simulação realística podem ser desenvolvidas sem perdas da eficiência em meio remoto.

\begin{tabular}{|c|c|c|}
\hline \multicolumn{3}{|c|}{$\begin{array}{l}\text { As atividades de simulação realística podem ser desenvolvidas sem perdas da eficiência em } \\
\text { meio remoto. }\end{array}$} \\
\hline Concordaram & 13 & $14.4 \%$ \\
\hline Neutros & 9 & $10.0 \%$ \\
\hline Discordaram* & 68 & $75.6 \%$ \\
\hline
\end{tabular}

*p $<0.0001$ Teste Qui-Quadrado Aderência. Fonte: Elaborado pelos autores.

É quase unanime afirmar que para os participantes deste estudo, é importante o retorno de $100 \%$ das atividades presenciais para melhorar a aprendizagem $92,2 \%(\mathrm{n}=83)$, mesmo resultado obtido quando perguntado se a pandemia dificultou a reunião de grupos para realização de atividades educacionais, pois, a forma virtual não substitui a presencial (Figura 4). A 
relação médico-paciente e docente-discente, facilita o feedback, a avaliação das habilidades práticas e, de acordo com o CNE as escolas devem providenciar o reordenamento pedagógico a partir do retorno das atividades presenciais (Brasil, 2014).

Figura 4 - Percepção docente sobre as dificuldades de realizar reuniões de estudo durante a pandemia e a importância do retorno das atividades presenciais.

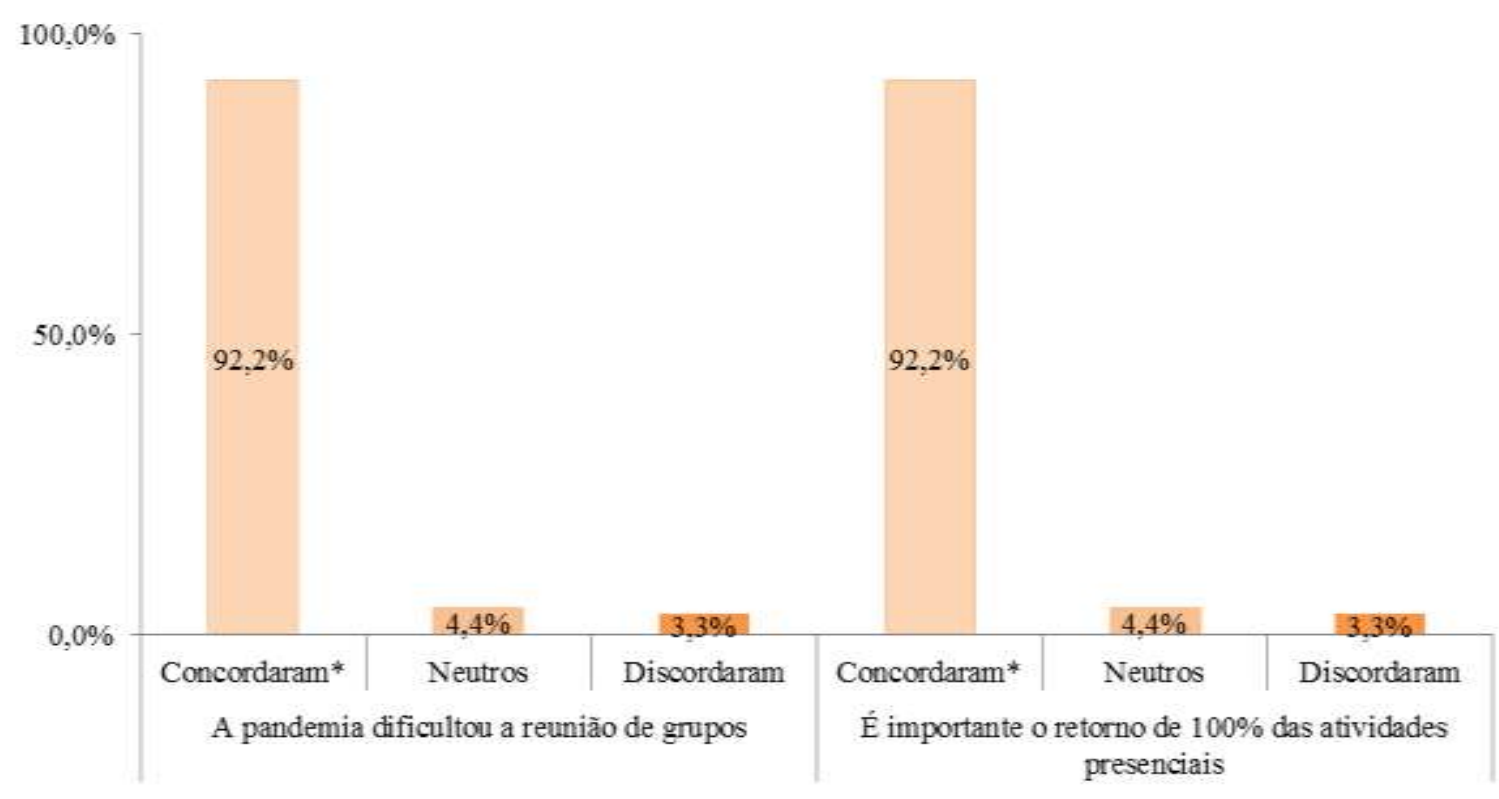

Fonte: Elaborado pelos autores.

Hodges et al. (2020) ao analisar "diferenças entre o ensino online e o ensino remoto de emergência", afirma que a atividade educacional de forma planejada não se compara em qualidade com a experiência educacional remota em momentos de crise, e que as metodologias aplicadas com sucesso dependem de uma série de fatores como tamanho e perfil da turma e dos objetivos do ensino pois existem limitações no ensino remoto que precisam ser consideradas.

Certas habilidades ou atitudes no ensino de saúde não são possíveis via ensino remoto, tais como habilidades sociais básicas (empatia, comunicação assertiva, entre outras) e habilidades comumente denominadas "técnicas" (Exame clínico, procedimentos assistenciais e/ou diagnósticos etc.). Outras variáveis a serem consideradas compõem as oportunidades de aprendizagem decorrentes da própria convivência dos estudantes na universidade, que promove não apenas o desenvolvimento de habilidades profissionais e interpessoais, mas também produz efeitos sobre a saúde física e mental dos estudantes (Gusso et al., 2020).

\section{Conclusão}

Considerando o objetivo deste estudo e os resultados que o alcançaram em uma amostra de conveniência, a maioria dos docentes respondentes ao questionário foi do gênero feminino, com diferença significativa entre a variável sexo, embora a maior dificuldade encontrada na adaptação para uso de ferramentas digitais tenha acontecido entre os docentes do sexo masculino. A média de idade dos participantes correspondeu há 43,9 anos, a maioria formada por docentes com formação profissional em Medicina, com tempo médio de formação de 19,6 anos, com título stricto sensu e média de 19 anos de experiência de trabalho docente. Percebeu-se que a maioria não teve dificuldades na adaptação para o uso de ferramentas virtuais, entretanto $92,2 \%$ deu importância ao treinamento institucional para adaptar metodologias ativas ao meio remoto, e está consciente de que a forma online gera desestímulo no discente. Todavia essas metodologias ativas podem ser adaptadas e 
contribuir com o aprendizado desde que adequadamente selecionadas, mas não substituem as aulas presenciais. Com base nisso, conclui-se que na percepção dos docentes deste estudo, o aprendizado por meio do ensino remoto é possível, em algumas áreas do conhecimento, desde que haja planejamento adequado aos objetivos de aprendizagem, mas, para a formação médica, a maioria dos docentes concordou com a necessidade do retorno às atividades presenciais.

\section{Referências}

Appenzeller, S., Menezes, F. H., Santos, G. G., Padilha, R. F., Sabino, H., \& Bragança, G. J. F. (2020). Novos Tempos, Novos Desafios: Estratégias para Equidade de Acesso ao Ensino Remoto Emergencial. Rev. bras. educ. med. 44: 04-20.

Bardin, L. (2011). Análise de conteúdo. (70a ed.), 229

Brasil. Ministério da Educação. (2014). Conselho Nacional de Educação. Parecer CNE/CES n 116 . Diretrizes curriculares nacionais do curso de graduação em medicina. Diário Oficial da União. 6 Jun 2014. p. 17

Brasil. Ministério da Educação. (2020). Portaria ${ }^{\circ}$ 343, de 17 de Março. Dispõe sobre a substituição das aulas presenciais por aulas em meios digitais enquanto durar a situação de pandemia do Novo Coronavírus - COVID-19. Diário Oficial da União.18 Mar 2020. p 39.

Burci, T. V. L., Santos, A. P. S., Mertzig, P. L. L., \& Mendonça, C. T. M. (2020). Ambientes virtuais de aprendizagem: a contribuição da educação a distância para o ensino remoto de emergência em tempos de pandemia. Revista de educação matemática e tecnologia iberoamericana. 11:1-16.

César, I. D., Danailof, K., \& Alves, F. K. (2020). Das aulas presenciais para o ambiente virtual: estratégias de ensino em saúde coletiva para alunos de graduação em saúde. Revista de Humanidades Digitais. 2:8-19.

Faria, C. D., \& Amaral, C. G. do. (2021). Educação Médica em tempos de pandemia e a utilização de metodologias ativas mediadas por tecnologia. Revista brasileira de educação médica. 45:076.

Fonseca, D. M. (2008). A pedagogia científica de Bachelard: uma reflexão a favor da qualidade da prática e da pesquisa docente. Educ. Pesqui. 34 : 15-29.

Fossa, R. da S., Benedetti, A. C., Esteves, P. E. do C. C., \& Silva, R. H. A. (2020). Ensino Remoto Emergencial em um curso de Medicina: avaliação do trabalho docente na perspectiva discente. Revista Docência do Ensino Superior. 10:1-21.

Gomes, V. T. S., Rodrigues, R. O., Gomes, R. N. S., Gomes, M. S., Viana, L. V. M., \& Santana, F. (2020). The Covid-19 Pandemic: Repercussions of Remote Education on Medical Training. Rev. bras. educ. med. 44: 52-71.

Gusso, H. L., Archer, A. B., Luiz, F. B., Sahão, F. T., Luca, G. G., Henklain, M. H. O., Panosso, M. G., Kienen, N., Beltramello, O., \& Gonçalves, V. M. (2020). Ensino Superior em Tempo de Pandemia: Diretrizes à Gestão Universitária. Educação \& Sociedade. 41:1-27.

Hodges, C., Trust, T., Moore, S., Bond, A., \& Lockee, B. (2020). Diferenças entre o aprendizado online e o ensino remoto de emergência. Revista da Escola, Professor, Educação e Tecnologia. 2:1-12.

Marsilli, L. R. B., Smecellato, F. B., \& Silva Júnior, O. C. (2020). Medical education in COVID-19 pandemic: medical students' point of view. Medicina. 53:490-4.

Meireles, M. A. C., Fernandes, C. C. P., \& Silva, L. S. (2019). Novas diretrizes curriculares nacionais e a formação médica: expectativas dos discentes do primeiro ano do curso de medicina da uma instituição de ensino superior. 43: 67-78.

Mello, J. B., Sampaio Neto, L. F., Geraldini, A. F. S., \& Cesaretti, M. L. R. (2021). Ensino Remoto em tempos de pandemia: a experiência da Pontifícia Universidade Católica de São Paulo (PUC-SP). Medicina (Ribeirão Preto). 54:184799.

Modelski, D., Giraffa, L. M. M., \& Casartelli, A. O. (2019). Tecnologias digitais, formação docente e práticas pedagógicas. Educ. Pesqui. 45:1-17.

Rios, I. C., Medeiros Junior, M. E., Fernandes, M. T. A., Zombini, E. V., Pacheco, M. K. O., Mascarenhas, E. G., \& Vital Junior, P. F. (2021). Mentoria virtual para estudantes de medicina em tempos de covid-19. Revista Brasileira de Educação Médica. 45:e170.

Rodrigues, B. B., Cardoso, R. J., Peres, C. H. R., \& Marques, F. F. (2020). Aprendendo com o imprevisível: Saúde mental dos universitários e educação médica na pandemia de Covid-29. Revista brasileira de educação médica. 45: e0149.

Santos, H. M. R. (2020). Os desafios de educar através da Zoom em contexto de pandemia: investigando as experiências e perspectivas dos docentes portugueses. Práxis Educativa. 15:1-7.

Santos, G. M. R. F., Silva, M. E., \& Belmonte, B. R. (2021). COVID-19: ensino remoto emergencial e saúde mental de docentes universitários. Rev. Bras. Saud. Mater. Infant. 21: 5245-5251.

Saraiva, K., Traversini, C., \& Lockmann, K. (2020). A educação em tempos de COVID-19: ensino remoto e exaustão docente. 15:1-24.

Schuhmacher, V. R. N., Alves Filho, J. P., \& Schuhmacher E. (2017). As barreiras da prática docente no uso das tecnologias de informação e comunicação. Ciênc. Educ. 23: 563-576.

Silva, P. H. S., Faustino, L. R., Sobrinho, M. S. O., \& Silva, F. B. F. (2021). Educação remota na continuidade da formação médica em tempos de pandemia: viabilidade e percepções. Revista Brasileira de Educação Médica. 45: e044.

b- Silva, V. C., Silva, Y. C. B., Mota, J. V. F., Moraes, F. C. A., Ramos, W. S., \& Neder, P. (2021). Desafios da implantação de aulas remotas no curso de medicina no mundo pós-pandemia. Revista Científica Multidisciplinar Núcleo do Conhecimento. 2:45-61. 